\title{
The surdo-cardiac syndrome and therapeutic observations
}

\author{
P. M. Olley and R. S. Fowler \\ From the Department of Cardiology, The Hospital for Sick Children, Toronto, Ontario, Canada
}

\begin{abstract}
First recognized in 1957, the surdo-cardiac syndrome includes congenital deafness, prolonged $Q T$ interval, and a high incidence of syncope and sudden death. Haemodynamic studies in two patients were normal except for an abnormal wave during left ventricular diastole probably related to abnormal left ventricular relaxation. The syncopal attacks are based on cardiac arrhythmias: both ventricular fibrillation and asystole may occur. Abnormal adrenergic stimulation of the heart is probably responsible. Propranolol appears to be effective in preventing the syncopal attacks. Artificial pacemaking provoked ventricular fibrillation in one patient and seems contraindicated.
\end{abstract}

Jervell and Lange-Nielson (1957) first described a syndrome characterized by congenital nerve deafness, prolongation of the QT interval in the electrocardiogram, syncopal attacks, and sudden death. This was later named the surdo-cardiac syndrome (Jervell, Thingstad, and Endsjö, 1966). The cause of the QT prolongation remains unknown. The syncopal attacks and sudden death probably result from cardiac dysrhythmias, but there have been few electrocardiograms obtained during attacks. Attacks first occur during early childhood, and until adolescence the outlook is grave; of 19 described cases, 8 have died suddenly in childhood. In those children surviving to adolescence the frequency of attacks diminishes and may cease completely. Any therapy which will protect these children through this hazardous period should be vigorously applied.

We wish to describe two further patients with this unusual syndrome, to report our haemodynamic findings, and to describe our experience in treating this condition.

\section{Case reports}

Case I A 5-year-old boy was found to be deaf at Io months of age. He was first admitted to hospital when 18 months old after two syncopal attacks. The first attack followed a trivial injury. He became pale and then lost consciousness for approximately ro minutes, during which time there were clonic limb movements. The second attack occurred two weeks later. He again became extremely pale, limp, and withdrawn. ConsciousReceived 16 December 1969. ness was not completely lost on this occasion. This attack lasted 20 minutes.

Physical examination at that time was completely normal except for deafness and the presence of a high-pitched early systolic murmur maximal in the fourth left interspace, suggesting a small ventricular septal defect. Subsequent examinations have not revealed any new findings.

Investigations on this first admission included full blood count and ESR, serum electrolytes, serum calcium and phosphorus, blood glucose, chest $x$-ray, and electroencephalogram. These were all normal. Serum viral agglutination tests were negative. The only abnormality detected was in the electrocardiogram which showed sinus rhythm, prolonged QT interval ( $0.64 \mathrm{sec}$.), and $T$ wave inversion in several leads (Fig. I).

He was discharged with the diagnosis of myocarditis, aetiology not determined. The diagnosis was subsequently revised to the surdo-cardiac syndrome.

He continued to have occasional attacks of syncope, and as he grew older it became clear that these were nearly always precipitated by fright, anger, or frustration. When the correct diagnosis. was recognized digoxin was tried over a 6-month period. It had no effect on the frequency of his attacks nor did it reduce their severity. Digoxin had no constant effect on the QT interval which varied but continued to be abnormally long.

When 3 years old he was readmitted for cardiac catheterization to exclude muscular subaortic stenosis. This investigation was completed without complications. On this admission his resting electrocardiogram showed a wandering pacemaker and occasional periods of nodal rhythm.

From this time his attacks increased in frequency, and by his $4^{\text {th }}$ birthday he was experiencing several severe attacks each week. He was 
readmitted and for the first time an electrocardiogram was obtained during a spontaneous attack. This showed basically asystole with short runs of nodal rhythm every 2 seconds, so-called periodic rhythm (Fig. 2). This dysrhythmia suggested the use of an artificial pacemaker and a temporary transvenous unit was inserted. We found that despite a resting heart rate of 60 a minute it was only possible to completely capture his heart at rates exceeding 100 a minute, using a $1.5 \mathrm{mV}$ stimulus. Not unexpectedly, after 48 hours of pacing he developed ventricular tachycardia progressing to ventricular fibrillation. Successful defibrillation was followed by attempts to reposition the catheter, which provoked further ventricular fibrillation (Fig. 3). Intravenous propranolol stabilized the heart rhythm. The pacemaker catheter was withdrawn.

He was then placed on propranolol $5 \mathrm{mg}$. 4 times a day, increasing to Io mg. 4 times a day, with a most gratifying response. His attacks have been reduced from 3 to 4 severe attacks a week to 2 minor attacks and I severe attack in 6 months. He remains well at the time of writing.

Case 2 In a 9-year-old boy, deafness was noted in infancy. He first developed seizures when aged I 8 months. From the beginning these attacks were precipitated by excitement, anger, or fright. $\mathrm{He}$ was referred to the Neuroconvulsive Clinic at The Hospital for Sick Children, Toronto, where initial and subsequent neurological examinations have been normal except for the deafness and associated speech deficit. Electroencephalograms have been repeatedly normal. His attacks were considered not to be typically epileptic. He was, however, followed in the Neuroconvulsive Clinic for some years, and was treated with varying combinations of phenobarbitone, primidone, phenytoin sodium. The frequency of his attacks was reduced but they were not abolished.

When 5 years old, he was first referred to the Cardiac Clinic because an early systolic murmur had been detected. Physical examination at that time was completely normal except for the deafness and a short early systolic murmur maximal at the left sternal border in the fourth space. The chest $x$-ray was normal. An electrocardiogram showed sinus rhythm with an abnormally long QT interval. He was diagnosed as having an innocent systolic murmur; the significance of the long QT interval was not recognized. The surdocardiac syndrome was diagnosed on his third visit to the Cardiac Clinic when he was 8 years old. He was admitted to hospital for cardiac evaluation in March 1969.

Physical examination was unchanged. The heart was not enlarged and the murmur was unaltered. The chest $x$-ray was normal. Blood count, serum electrolytes, and serum calcium were repeatedly normal. A repeat electroencephalogram was normal. The electrocardiogram showed sinus rhythm with a QT interval of $0.56 \mathrm{sec}$. (rate 72) and bizarre $T$ wave inversion (Fig. 4).

Cardiac catheterization was performed without complication.

His primidone and phenytoin sodium therapy

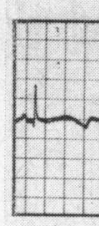

1

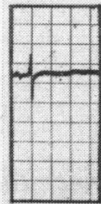

$\mathrm{RV}_{3}$

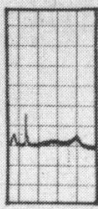

II

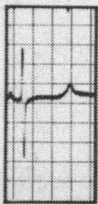

$V_{1}$

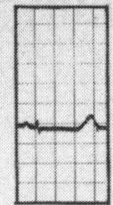

III

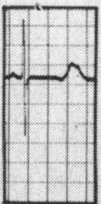

$\mathrm{V}_{2}$

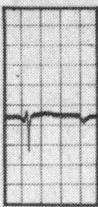

$A \vee R$

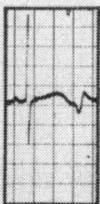

$V_{4}$

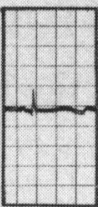

$A V L$

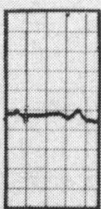

$V_{S}$

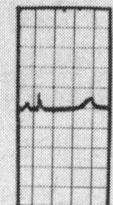

A VF

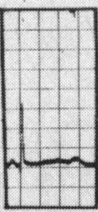

$V_{0}$
FIG. I Resting electrocardiogram. Case I (time lines $0.20 \mathrm{sec}$.).

was withdrawn, and he was monitored constantly over two separate one-week periods. No attacks occurred and no dysrhythmias were recorded. Discharge and return to school on no therapy resulted in a severe exacerbation of his seizures. Digoxin shortened the QT interval but did not prevent his attacks. Because phenobarbitone had previously controlled his attacks phenobarbitone $30 \mathrm{mg}$. three times a day was restarted with immediate control of his attacks.

He remains almost free of attacks on this therapy.

Neither patient has a family history of congenital deafness or syncopal attacks.

\section{Cardiac catheterization}

Haemodynamic studies were undertaken in these two patients to exclude muscular subaortic sten-

FIG. 2 Spontaneous attack showing periodic rhythm. Case I.

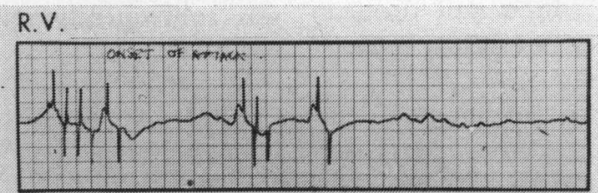

ONSET
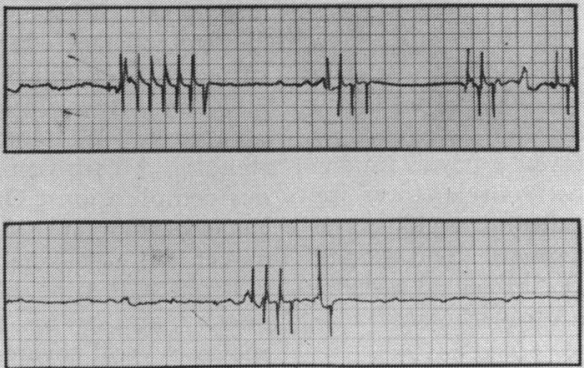


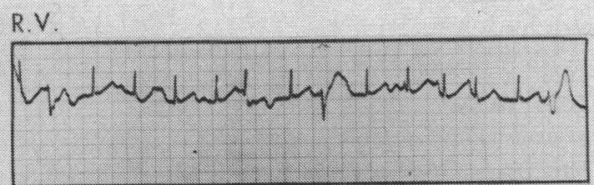

21 secs. after + Massage

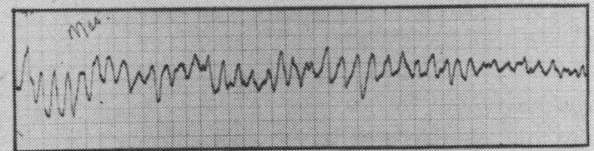

$45 \mathrm{sec}$. Mas

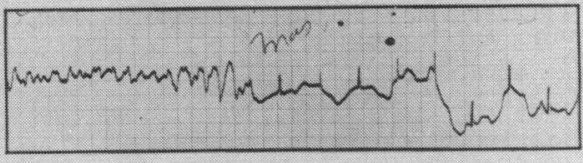

57 secs. Mas

60 secs

FIG. 3 Ventricular fibrillation during insertion of pacing catheter. Case $I$.

FIG. 4 Resting electrocardiogram. Case 2.

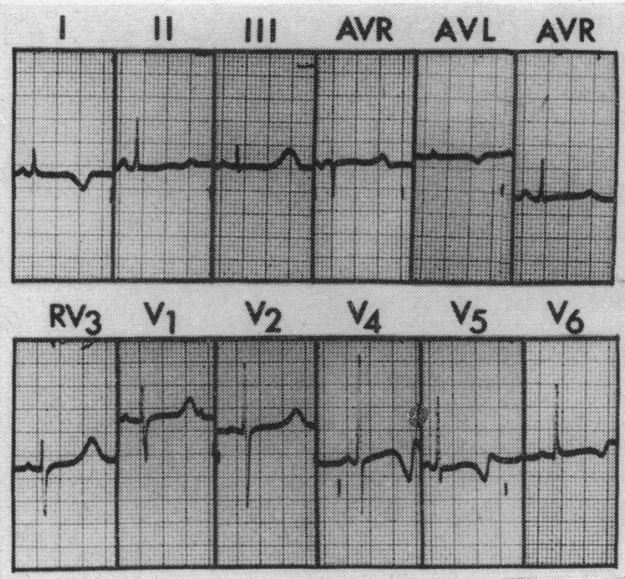

FIG. 5 Left ventricular pressure trace from

Case $I$.

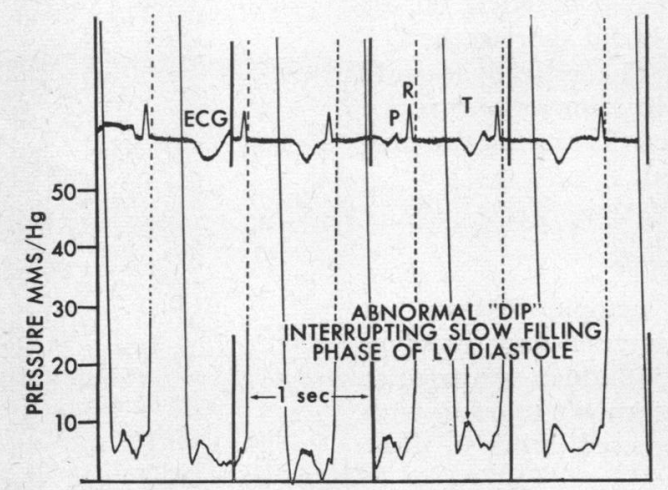

TABLE Haemodynamic findings in surdo-cardiac syndrome

\begin{tabular}{|c|c|c|c|c|}
\hline & \multicolumn{2}{|l|}{ Case I } & \multicolumn{2}{|l|}{ Case 2} \\
\hline & $\mathrm{O}_{2}$ satn. & $\begin{array}{l}\text { Pressure } \\
(\mathrm{mm} . \mathrm{Hg})\end{array}$ & $\mathrm{O}_{2}$ satn. & $\begin{array}{l}\text { Pressure } \\
(\mathrm{mm} . \mathrm{Hg})\end{array}$ \\
\hline Superior vena cava & 66 & - & 65 & - \\
\hline Inferior vena cava & 67 & - & 67.5 & - \\
\hline Right atrium & 67 & - & 63.5 & $\overline{4}$ \\
\hline Right ventricle & 67 & $20 / 0$ & 66 & $\begin{array}{l}4 \\
27 / 4\end{array}$ \\
\hline Pulmonary artery & 64 & $2 \mathrm{I} / 6 \overline{\mathrm{II}}$ & 69 & $27 / 9 \overline{18}$ \\
\hline Left atrium & 95 & - & - & $\frac{P A \text { wedge }}{12.5}$ \\
\hline Left ventricle & 97 & $102 / 5$ & 96 & $115 / 10$ \\
\hline Aorta & 97 & $102 / 50 \overline{58}$ & 96 & $105 / 64 \overline{82}$ \\
\hline
\end{tabular}

osis. This appears to be the first report of haemodynamic studies in the surdo-cardiac syndrome.

Oximetric and pressure data are summarized in the Table. The resting pressures were normal. Left ventricular and right ventricular end-diastolic pressures were normal. Intravenous infusion of isoprenaline hydrochloride failed to provoke a gradient across the left ventricular outflow tract. Left ventricular angiograms were normal in both patients. There was no suggestion of a ventricular septal defect in either patient.

The only unusual finding in both cases was the presence of a mid-diastolic dip in the left ventricular pressure trace during the slow filling phase (Fig. 5).

If the prolonged QT interval indicates delayed repolarization of ventricular muscle, perhaps this late fall in pressure observed during left ventricular diastole represents sudden increased left ventricular compliance or further ventricular relaxation as repolarization is completed.

\section{Discussion}

The original description of severe congenital bilateral nerve deafness, associated with prolonged QT interval, syncope, and sudden death, has been followed by several further reports. Fraser, Froggatt, and James (1964) made a notable contribution when they surveyed a large number of deaf children in Northern Ireland. They were able to add 9 new cases to the published reports. Their survey and others suggest that the over-all incidence of the surdo-cardiac syndrome is less than I per cent of all deaf children (James, 1967; Puletti, Jacobellis, and Borghi, 1967; Sánchez Cascos, Sánchez-Harguindey, and de Rábago, 1969). The evidence favours transmission as an autosomal recessive, and suggests that the responsible gene is largely confined to North European races.

Characteristically the attacks first occur in early childhood. They are often precipitated 
by fright or anger; while most individual attacks appear self-limiting, 44 per cent of published cases eventually had a fatal attack. These attacks result from cardiac dysrhythmia. In a related syndrome with all the features except deafness, ventricular fibrillation was recorded during a fatal attack (Ward, 1964). In our first case, at least one attack was due to asystole. In the same patient a pacemakerinduced attack was due to ventricular tachycardia which progressed to ventricular fibrillation. It seems probable to us that the dysrhythmia may vary from attack to attack in the same patient.

The electrocardiographic features are striking. Many of the published cases have had slow resting heart rates. Atrial dysrhythmias are not uncommon. One of our patients had frequent periods of a wandering pacemaker and at other times established nodal rhythm. The other patient has always exhibited sinus rhythm with a resting rate around 70 beats a minute. Both patients showed normal increases in heart rate on exercise.

The PR interval has always been normal during sinus rhythm. The QRS complexes are also within normal limits. The QT interval is strikingly abnormal. While generally greatly prolonged, it may vary very much, either spontaneously or in response to fright or excitement. On occasions the QT interval may be normal. In our first patient it varied from $0.44 \mathrm{sec}$. to $0.66 \mathrm{sec}$. (at rate $80 / \mathrm{min}$.), while in our second patient it has varied from $0.32 \mathrm{sec}$. to $0.68 \mathrm{sec}$. (rate 68-76). Digoxin and catacholamines may shorten the QT interval, but in view of the established variability of the QT interval we feel it is difficult to verify any such effect. However, one of our patients showed conspicuous shortening of the QT interval while receiving digoxin.

Bizarre and variable $T$ wave abnormalities also typify the surdo-cardiac syndrome. Deep symmetrical $T$ wave inversion may be present in several leads. Our patients have shown a beat-to-beat variation in their $T$ waves not attributable to the respiratory cycle.

The clinical features of the attacks and the electrocardiographic features of the syndrome seem well established. The basis of the electrocardiographic abnormality and the mechanism of the attacks remain speculative.

Increasing experimental work is linking the $Q T$ interval, the ST segment, and the $T$ wave of the electrocardiogram to certain adrenergic mechanisms. Specific areas of the mid-brain can be stimulated to produce changes in the ST segment and T wave. Asymmetrical sympathetic stimulation of the ventricle produces QT prolongation and gross $T$ wave abnor- malities (Yanowitz, Preston, and Abildskov, 1966). Simple aliphatic aldehydes related to more complex aldehydes which possess sympathomimetic properties can produce selective QT prolongation (James and Bear, 1968). The phenothiazine drug, thioridazine, alters the myocardial norepinephrine content. Clinically, QT prolongation and sudden death have been described in patients receiving this drug (Kelly, Fay, and Laverty, 1963). It is, therefore, attractive to relate the cardiac abnormalities of the surdo-cardiac syndrome to an abnormality of adrenergic neural control or of myocardial norepinephrine metabolism. Perhaps an abnormal enzyme system located at the cell membrane causes an abnormal response to neural or circulating norepinephrine. Maybe the final abnormality is one of ionic movements across the cell membrane.

The intrinsically slow heart rate coupled with the long QT interval causes an abnormally long vulnerable period, enhancing the possibility that ectopic beats will trigger a ventricular dysrhythmia.

Therapeutic observations Necropsy studies in this syndrome have reported a macroscopically and microscopically normal heart. Death is, therefore, likely to be due to electrical rather than to mechanical failure. Furthermore, there is good evidence suggesting that increasing age brings a decrease in frequency of attacks and that they eventually cease in late adolescence. The rarity with which a prolonged QT interval is found in deaf adults or in the parents of affected children suggests that the $Q T$ interval also returns to normal. For these reasons effective therapy in childhood is well worth while and every possibility should be explored.

\section{Digoxin}

This drug has been reported to shorten the QT interval and to reduce the severity and frequency of attacks. It had no effect on the attacks in our patients. It did shorten the QT interval in one of them. Theoretically it could increase the risk of dysrhythmia and syncope by producing ectopic activity during the prolonged vulnerable period.

\section{Quinidine and procainamide}

These drugs suppress ectopic rhythm but also lengthen the QT interval and therefore the vulnerable period. Sudden ventricular fibrillation and death are known to occur with quinidine. We have not used either of these drugs in our patients. 


\section{Artificial pacemakers}

There are no published reports of the use of pacemakers in this condition. We felt that the basically slow heart rate facilitated the development of ectopic rhythmias. It seemed reasonable to attempt to increase the heart rate artificially in an endeavour to suppress ectopic rhythm. We recognized the risk of producing dysrhythmias by this technique. We felt this procedure was especially worth while in our patient whose spontaneous attacks were basically asystole.

Even with ideal electrode placement, it proved unexpectedly difficult to pace the heart. Only at rates in excess of 100 a minute and using a stimulus of $\mathrm{r} .5 \mathrm{mV}$ were we able to suppress completely the patient's own intrinsic rhythm. Not unexpectedly, ventricular fibrillation occurred during pacemaking, and further attacks were produced by repositioning of the electrode catheter (Fig. 3).

On the basis of this meagre experience in a rare condition, we feel that artificial pacemaking is likely to be too hazardous for longterm use.

\section{Beta adrenergic blockade}

The highly suggestive links between the adrenergic system and the seizures of the surdo-cardiac syndrome naturally suggest the use of propranolol. Our first patient is now receiving this drug. It was introduced when he had been having three to four major attacks each week for several weeks. An initial dose of $5 \mathrm{mg} .4$ times a day was used in this 5year-old boy. Exhibition of propranolol coincided with a dramatic reduction of his attacks. For six weeks he was completely free of seizures. He then had two mild transient episodes. The dose was increased to ro $\mathrm{mg}$. 4 times a day. On this regimen he has had one major attack in six months. Our experience in this one case leads us to suggest beta adrenergic blockade as the first line of treatment.

\section{Phenobarbitone and phentytoin sodium}

While both these drugs act primarily on the central nervous system, both have some direct action on the myocardium in suppressing automaticity. We have already discussed the theory that the abnormalities in the surdocardiac syndrome may arise because of abnor- mal discharges from the midbrain. For both their direct action and their central nervous system effect these drugs appear worth a trial in this condition. Our second patient appears to be effectively controlled on phenobarbitone.

In treating syncopal attacks of the surdocardiac syndrome, we recommend that propranolol be tried first. If maximal doses fail to suppress the attacks, then phenobarbitone or phenytoin sodium, either in combination or separately, should be tried.

If these measures are ineffective, digoxin should be given a trial.

Because there is every hope that the children with this condition will eventually outgrow their attacks there is no reason to adopt a fatalistic attitude in the management of the surdo-cardiac syndrome.

\section{References}

Fraser, G. R., Froggatt, P., and James, T. N. (1964). Congenital deafness associated with electrocardiographic abnormalities, fainting attacks and sudden death. A recessive syndrome. Quarterly fournal of Medicine, 33, 361.

James, T. N. (1967). Congenital deafness and cardiac arrhythmias. American fournal of Cardiology, 19, 627.

- , and Bear, E. S. (1968). Cardiac effects of some simple aliphatic aldehydes. Fournal of Pharmacology and Experimental Therapeutics, 163, 300.

Jervell, A., and Lange-Nielsen, F. (1957). Congenital deaf-mutism, functional heart disease with prolongation of the Q-T interval and sudden death. American Heart fournal, 54, 59.

—, Thingstad, R., and Endsjö, T. (1966). The surdo-cardiac syndrome. American Heart fournal, 72, 582.

Kelly, H. G., Fay, J. E., and Laverty, S. G. (1963). Thioridazine hydrochloride (Mellaril): Its effect on the electrocardiogram and a report of two fatalities with electrocardiographic abnormalities. Canadian Medical Association fournal, 89, 546.

Puletti, M., Jacobellis, G. F., and Borghi, F. (1967). La sindrome di Jervell e Lange-Nielsen. Studio cardiologico di 2 I I sordomuti. Cuore e Circolazione, 5I, 25 I.

Sánchez Cascos, A., Sánchez-Harguindey, L., and de Rábago, P. (1969). Cardio-auditory syndromes. Cardiac and genetic study of 5 I I deaf-mute children. British Heart fournal, 3I, 26.

Ward, O. C. (1964). A new familial cardiac syndrome in children. Fournal of the Irish Medical Association, 54, 103.

Yanowitz, F., Preston, J. B., and Abildskov, J. A. (1966). Functional distribution of right and left stellate innervation to the ventricles: Production of neurogenic electrocardiographic changes by unilateral alteration of sympathetic tone. Circulation

C Research, 18, 416. 